\title{
NUMERICAL METHODS IN DIGITAL REAL-TIME SIMULATION*
}

\author{
BY \\ H. J. GRAY, JR. \\ University of Pennsylvania
}

1. Introduction. Under the sponsorship of the Office of Naval Research, Special Devices Center, the University of Pennsylvania conducted a study to determine the feasibility of building a digital real-time simulator for a system described by thirteen first-order non-linear differential equations. To achieve this, it was found necessary to improve either the speed of the machine [1] or the quality of the method of solution.

The present paper is an introductory one and deals with the latter problem and especially with the build-up of errors. Essentially the paper falls into two parts. A. Finding the characteristic equation associated with the difference equation by which the differential equation is replaced for purposes of numerical solution [2] B. Solution of the characteristic equation by conformal mapping. A diagram is given showing the method of solution in a case of practical interest.

2. Separation of round-off and truncation errors. This paper, being introductory in nature, will consider only linear constant-parameter systems of ordinary differential equations. Assume that the differential equations have been replaced by linear difference equations. Round-off errors may be treated as perturbations introduced at each step. Since the system is linear the propagating effect of a perturbation satisfies the same difference equations as the precisely computed solution and, if desired, the propagating effects of the individual perturbations may be summed and added to the precisely computed solution to obtain the actual computed solution.

3. Derivation of the characteristic equations. With the knowledge that the precisely computed solution and the round-off errors satisfy the same finite difference equation, it becomes important to study the properties of these difference equations.

Consider the system of linear constant-parameter differential equations

$$
d x_{i} / d t=\sum_{j=1}^{k} a_{i j} x_{j} ; \quad i=1,2, \cdots, k .
$$

Define the matrices

$$
\begin{aligned}
X & =\text { column }\left(x_{1}, x_{2}, \cdots, x_{k}\right), \\
A & =\left[\begin{array}{cccc}
a_{11} & a_{12} & \cdots & a_{1 k} \\
\cdot & \cdot & & \cdot \\
\cdot & \cdot & & \cdot \\
\cdot & \cdot & & \cdot \\
a_{k 1} & a_{k 2} & \ldots & a_{k k}
\end{array}\right] .
\end{aligned}
$$

For simplicity in exposition, $A$ will be assumed to have real elements and distinct characteristic values.

A class of quadrature methods which have proven to be useful in real-time simulation

*Received May 26, 1953. 
involve in one time interval, when applied to Eq. (1), the sequence of computations given below. (Methods $\left[O_{N M}, C_{R Q}\right]$ )

where

$$
\begin{aligned}
& O_{N M}: \quad\left(x_{i}\right)_{n}^{\prime}=\sum_{i=1}^{N} a_{i 0}\left(x_{i}\right)_{n-i}^{\prime \prime}+h \sum_{i=1}^{M} b_{i 0}\left(d x_{i} / d t\right)_{n-i}^{\prime}, \\
& C_{R Q}:\left(x_{i}\right)_{n}^{\prime \prime}=\sum_{i=1}^{R} a_{i c}\left(x_{i}\right)_{n-i}^{\prime \prime}+h \sum_{i=0}^{0-1} b_{i c}\left(d x_{i} / d t\right)_{n-i}^{\prime},
\end{aligned}
$$

$$
\left(d x_{i} / d t\right)_{n}^{\prime}=\sum_{i=1}^{k} a_{i i}\left(x_{i}\right)_{n}^{\prime} ; \quad i=1,2, \cdots, k .
$$

A constant time interval, $h$, is assumed and the $a_{i 0}, b_{i 0}, a_{i c}$, and $b_{i c}$ are assumed to be known constants as are $N, M, R$, and $Q$. The symbol $\left(x_{i}\right)_{j}$ denotes "the $i$ th variable $x$ evaluated at time $t=j h, j$ an integer." The primes and double primes serve only to identify the two classes of the numbers $\left(x_{i}\right)_{i}$, namely the results of the operations $O_{N M}$ and $C_{R 0}$.

Equations (2) when expressed in matrix form become

$$
\begin{aligned}
& O_{N M}: X_{n}^{\prime}=\sum_{i=1}^{N} a_{i 0} X_{n-i}^{\prime \prime}+h \sum_{i=1}^{M} b_{i 0} A X_{n-i}^{\prime}, \\
& C_{R Q}: X_{n}^{\prime \prime}=\sum_{i=1}^{R} a_{i c} X_{n-i}^{\prime \prime}+h \sum_{i=0}^{0-1} b_{i c} A X_{n-i}^{\prime},
\end{aligned}
$$

where $X_{i}^{\prime}, X_{i}^{\prime \prime}$ are column matrices with elements $\left(x_{i}\right)_{i}^{\prime},\left(x_{i}\right)_{i}^{\prime \prime}$ respectively.

Proceeding in standard fashion [3], assume

$$
X_{n}^{\prime}=\operatorname{Re} X_{0}^{\prime} \exp (n w), \quad X_{n}^{\prime \prime}=R e X_{0}^{\prime \prime} \exp (n w),
$$

where $X_{0}^{\prime}$ and $X_{0}^{\prime \prime}$ are column matrices having complex elements and $w$ is as yet an undefined complex number. Substitution of (4) into (3) yields, after elimination of $X_{0}^{\prime \prime}$,

$$
\begin{aligned}
\left\{\left[h \sum_{i=0}^{0-1} b_{i c} \exp (-j w) A\right]\left[\sum_{i=1}^{R} a_{i c} \exp (-j w)-1\right]^{-1}\left[\sum_{i=1}^{N} a_{i 0} \exp (-j w)\right]\right. \\
\left.+E-h \sum_{j=1}^{M} b_{i 0} \exp (-j w) A\right\} X_{0}^{\prime}=0^{\circ},
\end{aligned}
$$

where $E$ is the unit matrix and $0^{\circ}$ is the null matrix.

For a non-trivial solution of Eq. (5) to exist, the determinant of the quantity in the curly brackets must vanish. This determinant is found to be the characteristic determinant of the matrix $A$, with its characteristic values $\lambda$ equal to functions of exp $w$. Simplification of the rather formidable expression obtained in this way yields the characteristic equation

$$
z=\zeta[\alpha+\beta \zeta]^{-1},
$$

where $z=\lambda h, p^{i}=\exp (-j w)$ (see section 4$)$;

$$
\begin{aligned}
& \zeta=\left[1-\sum_{i=1}^{R} a_{i c} p^{i}\right]\left[\sum_{i=0}^{Q-1} b_{i c} p^{i}\right]^{-1} \\
& \alpha=\sum_{i=1}^{N} a_{i 0} p^{i}, \quad \beta=\sum_{i=1}^{M} b_{i 0} p^{i} .
\end{aligned}
$$


Similarly it can be shown that for repeated closures using $C_{R Q}, z=\zeta$ if $\left|b_{0 c} h \lambda\right|<1$; for $O_{N M}$ used alone, $z=[1-\alpha] \beta^{-1}$; and for the Runge-Kutta method [4],

$$
\exp w=\sum_{j=0}^{4} z^{i} / j !
$$

4. Solution of the characteristic equations. Detailed specifications on the quality of simulation will not be given here. However, the accuracy requirements are generally considerably looser in simulation than in other scientific computations such that the conformal mapping method of solution given below has been found to be entirely adequate.

Any general solution $x_{i}$ of Eq. (1) may always be written as a linear combination of the functions $\exp \left(\lambda_{i} t\right), i=1,2, \cdots, k$ where the $\lambda_{i}$ are the (distinct) characteristic values of the matrix $A$. The $\lambda_{i}$ are called the "complex frequencies" or sometimes just "frequencies" of the system described by Eq. (1). By exploiting the parallel nature of the theories of linear constant-coefficient differential and difference equations, it is possible to define and talk about the "frequencies in the computed solution". Hence, determination in the computed solution of the frequency having the largest real part enables a determination of at least whether or not a given computed solution of Eq. (1) will decay to zero (stable) or grow indefinitely (unstable). This is the source of the name "stability charts" given to the conformal maps to be derived.

Equation (7) yields as roots, given an unique value of $z$, a set of values of $w$ differing by integer multiples of $2 \pi i$. However, (see Eq. 4) since exp $n(w+2 \pi i l)=\exp n w$, $n$ and $l$ being integers, it is sufficient to consider only one value of $w$. That value satisfying the inequalities $-\pi<\operatorname{Im} w \leq \pi$ will be chosen and will still be denoted by the symbol $w$ in the following.

Let

$$
w=\mu h .
$$

Equation (8) defines $\mu$, the frequency in the computed solution corresponding to $\lambda$, the frequency in the true solution.

Equation (7) being in a form in which it can be considered solved will not be discussed further. The remaining characteristic equations are written in the form $z=f(w)$. However, they may be put into the form

$$
\begin{aligned}
A_{0} r^{n}+A_{1} r^{n-1}+\cdots+A_{n} & =0, \\
A_{0} & \neq 0, \\
r^{i} & =p^{-i}=\exp j w,
\end{aligned}
$$

where the $A_{i}$ are first degree polynomials in $z=\lambda h$. Corresponding to a chosen value of $\lambda$, Eq. (9) has $n$ roots $r$, which when arranged in order of decreasing magnitude are [5] $r_{1}, r_{2}, \cdots, r_{n}$.

Since $w=\log _{e} r$, one has $w_{1}, w_{2}, \cdots, w_{n}$ where $\operatorname{Re} w_{1} \geq \operatorname{Re} w_{2} \geq \cdots \geq \operatorname{Re} w_{n}$ and $-\pi<\operatorname{Im} w_{i} \leq \pi$.

It is evident from the remarks made earlier about stability that $w_{1}$ is of interest.

A number of methods of finding $w_{1}$, given $\lambda$, were investigated including numerical extraction of roots and expansion of roots in a power series in $\lambda h$ but these were discarded in favor of the conformal mapping method for the following reasons. 
(a) $w_{1}$ as furnished by the conformal maps (stability charts) is sufficiently accurate for the simulation application.

(b) The computation required to prepare a stability chart is relatively routine and the amount of it is not much greater than that required for one root extraction by standard methods. Furthermore, the stability chart effectively solves all root extractions of practical interest for the given quadrature method.

(c) The stability charts furnish a convenient means of comparing the merits of the different quadrature methods.

In order to simplify the exposition, the following assumptions will be made concerning the nature of the characteristic equation, $z=f(w)$.

(a) $z$ is a single-valued analytic function of $w$ except at a finite number of poles.

(b) $w$ is a multi-valued function of $\mathrm{z}$. The restriction $-\pi<\operatorname{Im} w \leq \pi$ makes $w$ assume only a finite number of values for any one value of $z$.

The stability charts are obtained by mapping the $w$ plane into the $z$ plane using the characteristic equations. Essentially what this does is to determine one root for each $z$. Whether or not this is the root, $w_{1}$, is determined as follows: given $z=f(w)$ it is well known that there may exist for a fixed value of $z=z_{1}$, a root $w_{0}$ which has the same real part as $w_{1}$. The trio of points $z_{1}, w_{1}, w_{s}$ will be defined to be a "point on a branch contour". Hence, a "branch contour" will be the locus, if it exists, of "points on a branch contour" in both the $z$ and $w$ planes. Furthermore, every branch point lies on at least one branch contour.

Fix $z=z_{1}^{0}$ and determine the roots $w$ of $f(w)=z_{1}^{0}$ (assume that all the roots are distinct). Denote the point in the $w$ plane defined by the $w_{1}$ as $w_{1}^{0}$.

It can be stated that if a point $w^{*}$, which lies inside a simply connected region containing $w_{1}^{0}$ but not containing any points on a branch contour, is mapped into the $z$ plane by $z^{*}=f\left(w^{*}\right)$ then one root of $z^{*}=f(w)$ is $w^{*}$, and for all other roots $w_{s}$, Re $w_{s}<\operatorname{Re} w^{*}$.

This follows directly from the definition of "points on a branch contour" and the theorem [5] that the roots $w$ of $z=f(w)$ are continuous functions of $z$.
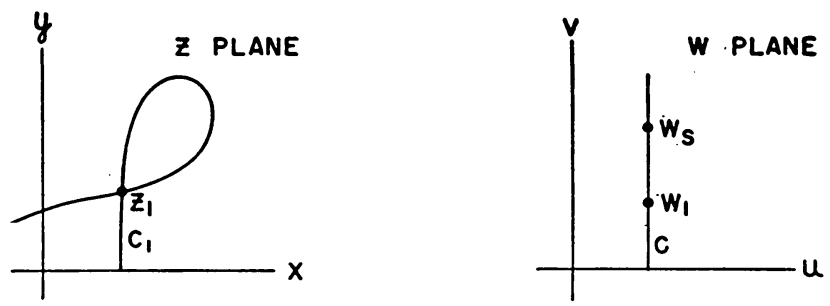

Frg. 1. Determination of points on a branch contour

To find points on the branch contours, let $w=u+i v$ follow $C$ in Fig. 1. Its image $z=x+i y$ may follow $C_{1}$. The crossover point $z_{1}$ and its images on $C$ lie on a branch contour by definition.

The characteristic equation, $z=f(w)$, for $\left[O_{41}, C_{41}\right]$ with coefficients given in (10) is mapped in the rough in Fig. 2. The contours in the $w$ plane are the $u=$ constant lines, with $v$ (and $u$ ) taken at intervals of 0.1 . Symmetry and periodicity restrict the required 


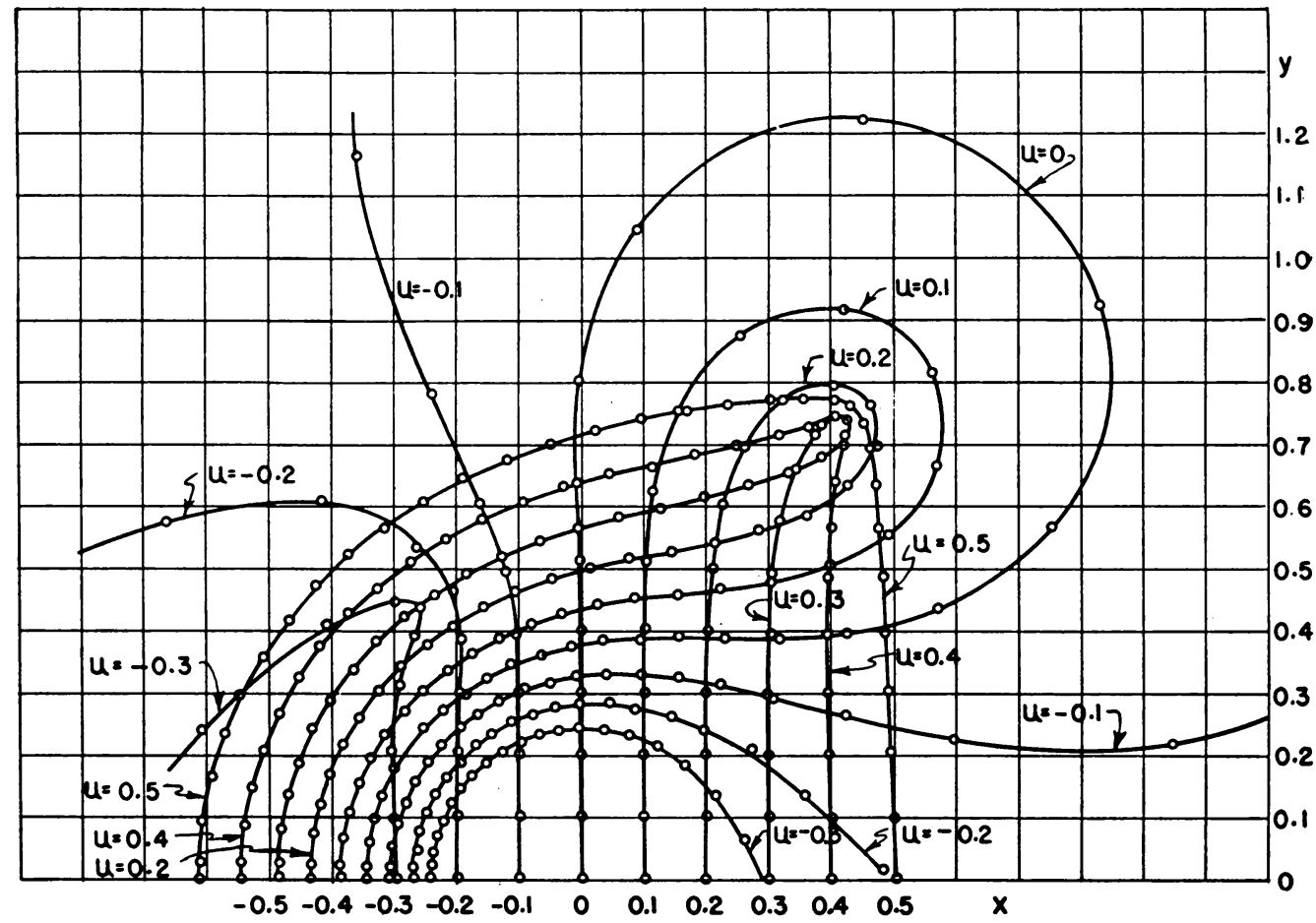

FIG. 2. Preliminary mapping for $\left[\mathrm{O}_{41}, \mathrm{C}_{41}\right]$

range of $v$ to $0 \leq v \leq \pi$. For $z=0$, it is found that $w_{1}^{0}=0$. The $w$ plane maps into the $z$ plane five times, but since only the dominant root, $w_{1}$, is of interest, four of the five coverings of the $z$ plane have been discarded (some of them in the sense of not even being computed) to obtain Fig. 3.

$$
\begin{aligned}
O_{41}: a_{10} & =-10 / 3 & C_{41}: & a_{1 c}=48 / 25 \\
a_{20} & =6 & a_{2 c} & =-36 / 25 \\
a_{30} & =-2 & a_{3 c} & =16 / 25 \\
a_{40} & =1 / 3 & a_{4 c} & =-3 / 25 \\
b_{10} & =4 & b_{0 c} & =12 / 25
\end{aligned}
$$

5. Computations. One way of using the charts is as follows. Given the system of differential equations, determine the frequencies, $\lambda$, in the system. Spot the points $z=\lambda h$ on the stability chart for the quadrature method to be used and read off the values of $w=\mu h$, a procedure rigorously correct only for linear constant-parameter systems.

The process will be illustrated by formal application to the linear varying-parameter system described by

$$
\begin{aligned}
& d x / d t=y \\
& d y / d t=-3 y-\left(6.25+2.4 t+0.36 t^{2}\right) x+f(t)
\end{aligned}
$$




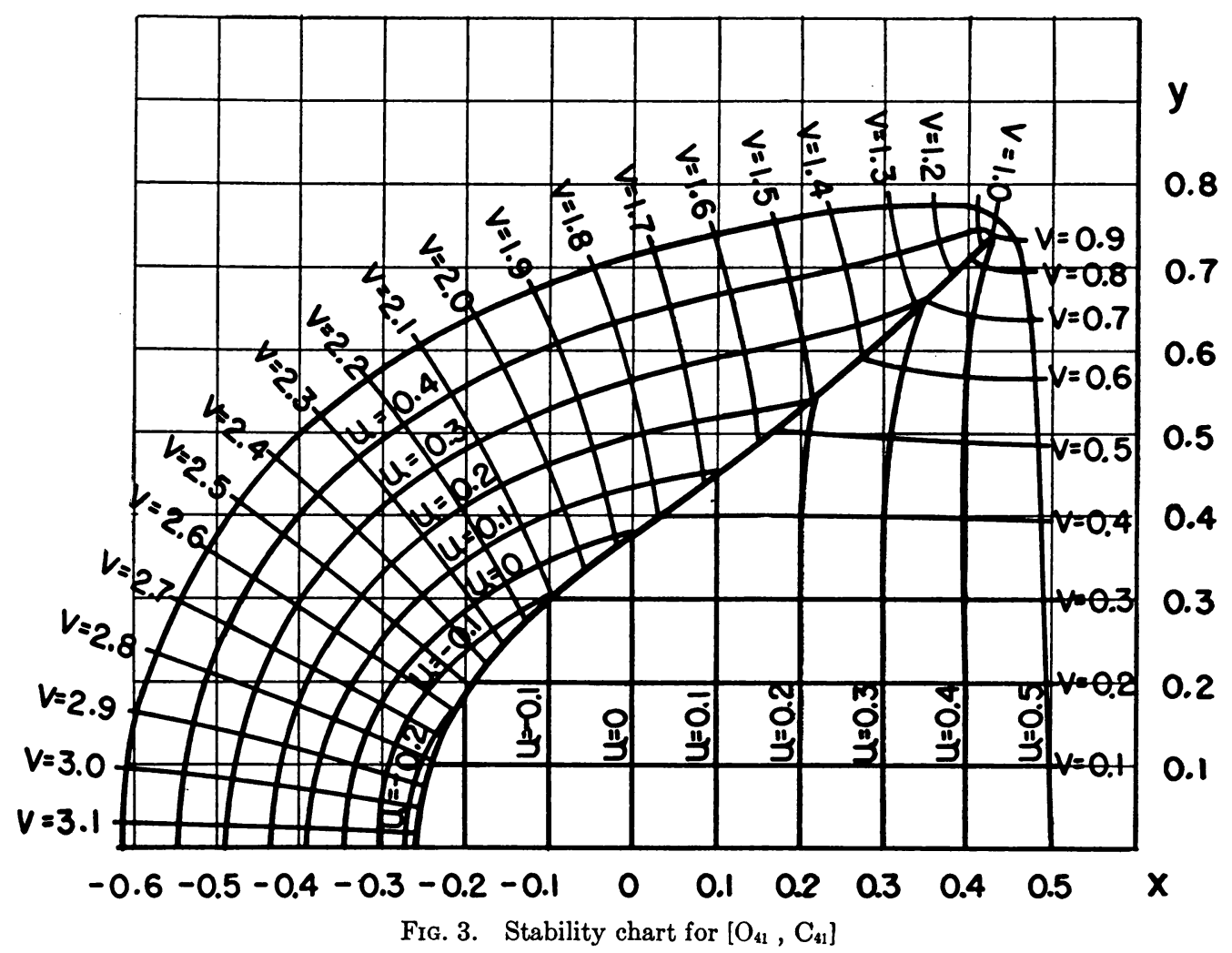

whose solution was computed for two cases as follows using method $\left[O_{41}, C_{41}\right]$ (Eq. 10).

(a) $h=0.1, f(t)= \pm 0.000 x x x$ where the $x x x$ were random numbers, and $x_{i}=1, y_{i}=1.5$ for $j=0, \cdots,-3$. The results are given by the $h=0.1$ points of Fig. 4.

(b) $h=0.02, f(t)=0$ and starting conditions as in (a) above. The results are given by the $h=0.02$ curve of Fig. 4 and serve to approximate the true solutions of Eq. (11).

The matrix $A$ is

$$
A=\left[\begin{array}{cc}
0 & 1 \\
-\left(6.25+2.4 t+0.36 t^{2}\right) & -3
\end{array}\right],
$$

and its characteristic values are

$$
\lambda=-1.5 \pm i(2+0.6 t) .
$$

For $h=0.02, z=-.03 \pm i(.04+0.012 t)$ which for $t \leq 5$ yields nearly the same value for $w$ (Fig. 3). However, for $h=0.1, z=-0.15 \pm i(0.2+0.06 t)$ crossing the $u=0$ line at $t=2$. The expected unstable behavior appears shortly thereafter (Fig. 4).

6. Conclusions. The introduction to a systematic and convenient method is described for determining the stability of a system by operating on the frequencies of the 


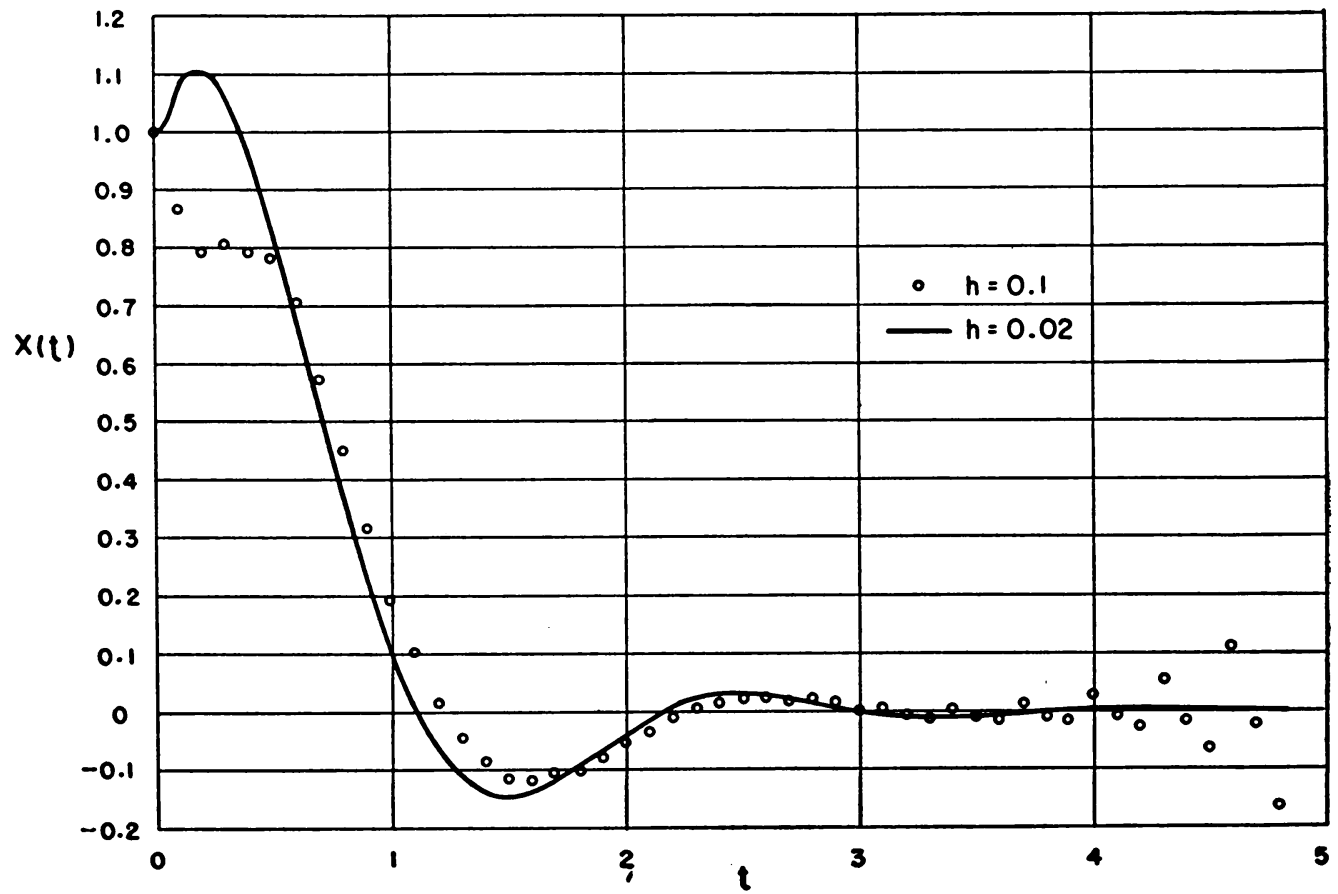

Fig. 4. Computations with $\left[\mathrm{O}_{41}, \mathrm{C}_{41}\right]$

system. The procedure is rigorously established for linear constant-parameter systems only, even though an example is given for a linear varying-parameter system.

7. Acknowledgement. The author wishes to acknowledge with thanks the assistance and many valuable suggestions given by Dr. Morris Rubinoff and Mr. C. T. Leondes of the University of Pennsylvania and Dr. F. J. Murray of the Columbia University Mathematics Department. This work was done under Contracts N6onr-24915 and Nonr-551(02) sponsored by the Office of Naval Research, Special Devices Center, Port Washington, New York, and is part of a thesis submitted in partial fulfillment of the requirements of the degree of Doctor of Philosophy in Electrical Engineering at the University of Pennsylvania, June 1953.

\section{APPENDIX}

The stability chart for $\left[O_{41}, C_{41}\right]$ together with charts for thirty-two other quadrature methods was computed directly from Eqs. 6 on the automatic digital computer at the University of Pennsylvania Computing Center. Specifically, for $\left[O_{41}, C_{41}\right]$, one has

$$
\begin{aligned}
\alpha & =-10 / 3 p+6 p^{2}-2 p^{3}+1 / 3 p^{4} \\
\beta & =4 p \\
\zeta & =\left[1-48 / 25 p+36 / 25 p^{2}-16 / 25 p^{3}+3 / 25 p^{4}\right][12 / 25]^{-1}, \\
z & =\zeta[\alpha+\beta \zeta]^{-1} ; \quad p=\exp (-w) .
\end{aligned}
$$




\section{REFERENCES}

1. H. J. Gray, Jr., The organization of a digital real-time simulator, Convention Record of the Institute of Radio Engineers Part 1-Radar and Telemetry, 85, March 23-26 (1953).

2. W. E. Milne, Numerical solution of differential equations, John Wiley and Sons, Inc., New York, 1953. This book contains an excellent bibliography and discussion on the subject.

3. L. M. Milne-Thomson, The calculus of finite differences, MacMillan and Co., 1933, p. $384 \mathrm{ff}$.

4. J. B. Scarborough, Numerical mathematical analysis, Johns Hopkins Press, 1950, p. 302.

5. Morris Marden, The geometry of the zeroes of a polynomial in a complex variable, American Math. Society Survey III, New York, 1949. 